\title{
DITERPENES FROM THE BARK OF JUNIPERUS CHINENSIS
}

\author{
Jim-Min Fang, Yi-Chien Sou, Yu-Hung Chiu and Yu-Shia Cheng* \\ Department of Chemistry, National Taiwan University Taipei, Taiwan 10617, Republic of China
}

(Received in revised form 27 April 1993)

Key Word Index Juniperus chinensis; Cupressaceae; bark; diterpenes; labdane-type; abietane-type; totarane-type; secoabietane; sandaracopimaric acid.

\begin{abstract}
Eighteen diterpenes were isolated from the bark of Juniperus chinensis. The new compounds include the $E$ and $Z$-isomers of 12,15-dihydroxylabda-8(17),13-dien-19-oic acid, the $E$ - and $Z$-isomers of $2 \alpha$-hydroxycommunic acid as well as 15,16-bisnor-8,17-epoxy-13-oxolabd-11E-en-19-oic acid, whose structures were determined by spectral methods. An epoxy communic acid, 12,13-epoxylabda-8(17),14-dien-19-oic acid and the isomer of dihydroxy communic acid, 12,13-dihydroxylabda-8(17),14-dien-19-oic acid, were isolated for the first time from a natural source.
\end{abstract}

\section{INTRODUCTION}

Juniperus chinensis is a common ornamental tree [1]. The compounds hinokiflavone and kayaflavone have been previously reported in this plant $[2,3]$. We recently isolated 13 lignans and 16 diterpenes in addition to other components from the leaves $[4,5]$. The diterpenes include semperviranes, abietanes, totaranes and a new chinanetype having isopropyl groups at the C-12, C-13, C-14 and $\mathrm{C}-11$ positions, respectively [5]. We report here 18 diterpenes, including labdanes, abietanes and totaranes, found in the bark of $J$. chinensis.

\section{RESULTS AND DISCUSSION}

The acetone soluble part of the bark of $J$. chinensis kaizuka was extracted with ethyl acetate and the extract subjected to chromatography to give the diterpene components. The known compounds were readily identified as sandaracopimaric acid (1) [6, 7], ferruginol (2) [8], 7dehydroabietanone (3) [9], sugiol (4) [7], 6 -hydroxy-7oxoferruginol (5) [10], 6,7-secoferruginol-6,7-dial (6) $[7$, $11]$, totarol (7) [5, 12], totarolone (8) [13], communic acid $(9$, cis/trans $=1: 3)[14,15]$, isocupressic acid $(10)[15,16]$ and agathic acid (11) $[17,18]$ by analyses of their physical and spectral properties (mp, [ $\alpha]$, IR, mass, ${ }^{1} \mathrm{H}$ and ${ }^{13} \mathrm{C}$ NMR spectra). Sandaracopimaric acid was the predominant component.

The IR spectrum of 12a showed the presence of carboxylic acid. The ${ }^{1} \mathrm{H}$ NMR spectrum (Table 1) revealed some structural characteristics such as a moiety of allylic alcohol $\mathrm{R}_{2} \mathrm{C}=\mathrm{CHCH}_{2} \mathrm{OH}$ appearing at $\delta 4.09(d)$ and 5.33 $(t)$, a secondary alcohol appearing at $\delta 4.05(d d)$, an exocyclic double bond appearing at $\delta 4.68(s)$ and $4.85(s)$,

\footnotetext{
*Author to whom correspondence should be addressed.
}

as well as three methyl groups appearing at $\delta 1.59(s), 1.17$ (s) and $0.63(s)$. By analysis of the ${ }^{13} \mathrm{CNMR}$ spectrum of $12 \mathbf{a}$ and comparison with the literature [19], 12a is assigned as 12,15-dihydroxylabda-8(17),13E-dien-19-oic acid. Compound 12a was dextrorotatory, $[\alpha]_{D}+33^{\circ}$, whereas the reported compound isolated from Guizotia scaba (Heliantheae) [19] is laevorotatory, $[\alpha]_{\mathrm{D}}-29^{\circ}$. By analogy to the structures of other chemical constituents such as $\mathbf{1 0}$ and 11, compound $12 \mathrm{a}$ is probably a labdane diterpene having the $(4 S, 9 S, 10 R, 12 S)$-configuration, whereas the previously reported compound (absolute configuration unknown) is the enantiomer of $12 \mathbf{a}$. The ${ }^{13} \mathrm{CNMR}$ resonances of $\mathbf{1 2 b}$ were very close to the values of the corresponding signals of 12a (Table 2), except for a methyl resonance $(\mathrm{C}-16)$ of $\mathbf{1 2 b}$ appearing at lower field $(\delta 17.0)$. A NOE experiment by irradiating the corresponding methyl group at $\mathrm{C}-13(\delta 1.67)$ caused $19 \%$ enhancement of $\mathrm{H}-14(\delta 5.41)$. Thus $12 \mathrm{~b}$ is assigned as $12,15-$ dihydroxylabda-8(17), 13Z-dien-19-oic acid, a 13Z-isomer of $12 \mathbf{a}$.

Compound 13a had an exact mass at $m / z 318.218$ attributable to a molecular formula $\mathrm{C}_{20} \mathrm{H}_{30} \mathrm{O}_{3}$. The IR spectrum showed characteristic absorptions of carboxylic acid. The UV absorption at $227 \mathrm{~nm}$ indicated the presence of a conjugated diene. The resonances of the corresponding olefinic protons appeared at $\delta 5.37(t), 6.30(d d, J$ $=17,11 \mathrm{~Hz}), 4.87(d, J=11 \mathrm{~Hz})$ and $5.03(d, J=17 \mathrm{~Hz})$ in addition to a proton $(\mathrm{H}-2)$ geminal to a hydroxyl group occurring at $\delta 4.13$ (dddd, $J=12,12,4,4 \mathrm{~Hz}$ ). Irradiation of the signal of the Me-10 group caused an NOE effect on $\mathrm{H}-2$, supporting the axial orientation of $\mathrm{H}-2$. The ${ }^{1} \mathrm{H}$ and ${ }^{13} \mathrm{C}$ NMR spectra of 13a and $b$ were similar except for the signals of C-14 and C-16. By comparison with the spectra of trans- and cis- communic acids $[14,20], 13 a$ and $b$ are assigned as $2 \alpha$-hydroxy-trans-communic acid and $2 \alpha$ hydroxy-cis-communic acid, respectively. Compound 13b having the $12 E$-configuration showed $\mathrm{H}-12$ at higher field 
<smiles>C=C[C@@]1(C)C=C2CC[C@]3(C(=O)O)[C@@H](C)CCC[C@]3(C)[C@]2(C)CC1</smiles>

1<smiles>CC(C)c1cc(C(C=O)C=O)c([C@@]2(C)CCCC(C)(C)C2(C)C)cc1O</smiles>

6

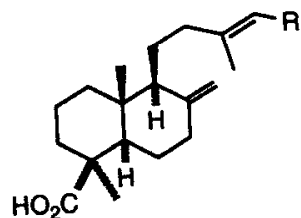

$10 \mathrm{R}=\mathrm{CH}_{2} \mathrm{OH}$

$11 \mathrm{~A}=\mathrm{CO}_{2} \mathrm{H}$<smiles>CC(C)c1cc2c(cc1O)[C@]1(C)CCC[C@H](C)[C@]1(C)CC2</smiles><smiles>CC(C)c1c(O)ccc2c1CC[C@H]1C(C)(C)C(=[V])CC[C@@]21C</smiles>

$7 \mathrm{Y}=\mathrm{H}, \mathrm{H}$

$8 Y=0$

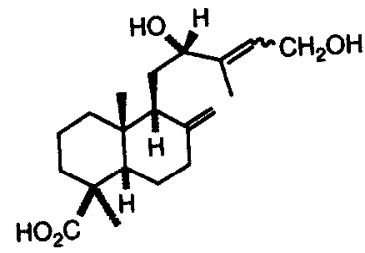

$12 a(E)$

$12 b(Z)$<smiles>[R]c1cc2c(cc1C(C)C)C(=O)C([R])[C@@]1(C)[C@@H](C)CCC[C@]21C</smiles>

$3 R^{1}=R^{2}=H$

$4 R^{1}=H, R^{2}=O H$.

$5 R^{1}=R^{2}=O H$

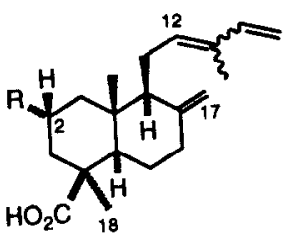

$9 R=H$

13a ( $(E), R=\mathrm{OH}$

$13 b(Z), R=O H$

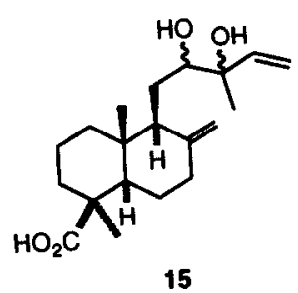

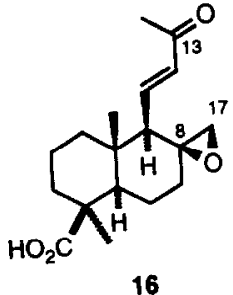<smiles>C=C[C@]12C[C@@H]3CC[C@H]4[C@](C)(C(=O)O)CCC[C@]4(C)[C@H]3C[C@H]1O[C@H]2C</smiles>

14

$(\delta 5.26)$ and $\mathrm{H}-14$ at lower field $(\delta 6.75)$ compared to the corresponding resonances of $13 \mathbf{a}$.

An epoxy communic acid, 12,13-epoxylabda-8(17),14dien-19-oic acid (14) was isolated. The methyl ester of 14 has been previously synthesized by epoxidation of the methyl ester of trans-communic acid [21,22]. The constituents of the bark of $J$. chinensis also include diastereomers of dihydroxy communic acid, 12,13dihydroxylabda-8(17),14-dien-19-oic acid (15). The $(12 R, 13 S)$-isomer $15 \mathrm{a}$ was purified, whereas the $(12 R$, $13 R)$-isomer $15 \mathrm{~b}$ was contaminated with $(12 S, 13 R S)$ isomers. The stereochemistry was determined by detailed analysis of their ${ }^{1} \mathrm{H}$ NMR spectra. The two $\mathrm{C}-17$ protons in the $(12 R)$-isomer $15 \mathrm{a}$ showed at $\delta 4.42$ and $4.82(\Delta \delta$ $=0.4 \mathrm{ppm})$, the corresponding protons in $15 \mathrm{~b}$ appeared at $\delta 4.43$ and $4.84(\Delta \delta=0.4 \mathrm{ppm})$. According to the literature $[22,23]$, the two $\mathrm{C}-17$ protons in the $(12 S)$-isomer showed a smaller difference in chemical shifts $(4.89-4.75$ $=0.14 \mathrm{ppm}$ ). The dihydroxylabdanoic acids $12 \mathrm{a}, \mathrm{b}$ having the (12S)-configuration also exhibited small chemical shift differences between the two $\mathrm{C}-17$ protons $(\Delta \delta<0.2 \mathrm{ppm}$, see Table 1). The methyl esters of 15a, b have been previously prepared by hydrolysis of the methyl ester of the epoxy communic acid $[21,22]$. The $\mathrm{H}-$ 16 in 15a appeared at higher field $(\delta 1.25)$ than that of $15 \mathrm{~b}$ $(\delta 1.30)$ [22].

The ${ }^{13} \mathrm{CNMR}$ spectrum of 16 showed 18 carbon signals, and the exact mass at $m / z \quad 306.182$ led to a molecular formula $\mathrm{C}_{18} \mathrm{H}_{26} \mathrm{O}_{4}$. The resonance at $\delta 180(s)$ was attributable to the carbon of a carboxylic acid, the resonances at $\delta 140(d), 136(d)$ and $197(s)$ were ascribed to $\mathrm{CH}=\mathrm{CH}-\mathrm{C}=\mathrm{O}$ of a conjugated ketone. The ${ }^{1} \mathrm{H}$ NMR spectrum showed a resonance at $\delta 2.19(s, 3 \mathrm{H})$ attributable to a methyl ketone. Compound 16 is assigned as 15,16-bisnor-8,17-epoxy-13-oxolabd-11E-en-19-oic acid. Two oxirane protons appeared at $\delta 2.56$ and 2.74 , which showed no NOE by irradiation of the Me-10 signal at $\delta 0.89$. Thus, the $\mathrm{C}-8 / \mathrm{O}$ bond is considered to have the 
Table 1. ${ }^{1} \mathrm{H}$ NMR spectral data of compounds $12 \mathrm{a}, \mathrm{b}, 13 \mathrm{a}, \mathrm{b}$ and $16\left(\mathrm{CDCl}_{3}\right.$ solution, $\delta$ values in $\mathrm{ppm}, J$ values in $\left.\mathrm{Hz}\right)$

\begin{tabular}{|c|c|c|c|c|c|}
\hline $\mathbf{H}$ & 122 & $\mathbf{1 2 b}$ & $13 \mathbf{a}$ & $13 \mathbf{b}$ & 16 \\
\hline 1 & $\begin{array}{l}1.03 \text { ddd }(13,13,4), 1.8 \\
m\end{array}$ & $1.04 \mathrm{~m}, 1.81 \mathrm{~m}$ & $\begin{array}{l}1.05 d d(12,12) \\
2.39 d(12)\end{array}$ & $\begin{array}{l}1.05 d d d(12,12,3) \\
2.39 d d(12,4)\end{array}$ & $1.05 \mathrm{~m}, 1.48 \mathrm{~m}$ \\
\hline 2 & $\begin{array}{l}1.43 \mathrm{~m}, 1.90 \mathrm{ddd} \\
(10,4,1)\end{array}$ & $1.48 \mathrm{~m} .190 \mathrm{~m}$ & 4.15 dddd $(12,12,4,4)$ & 4.13 dddd $(12,12,4,4)$ & $1.47 \mathrm{~m}, 1.89 \mathrm{~m}$ \\
\hline 3 & $\begin{array}{l}1.03 d d d(13,13,4) \\
2.09 \mathrm{~m}\end{array}$ & $1.04 \mathrm{~m}, 2.12 \mathrm{~m}$ & $\begin{array}{l}1.05 d d(12,12) \\
2.15 d d(12,4)\end{array}$ & $\begin{array}{l}1.05 d d(12,12) \\
2.15 d d(J 12,4)\end{array}$ & $1.06 \mathrm{~m}, 2.1 \mathrm{~m}$ \\
\hline 5 & $1.32 d d(11,4)$ & $1.33 \mathrm{~m}$ & $1.31 \mathrm{~m}$ & $1.28 \mathrm{~m}$ & $1.25 \mathrm{~m}$ \\
\hline 6 & $1.82 \mathrm{~m}, 1.95 \mathrm{~m}$ & $1.86 \mathrm{~m}, 1.97 \mathrm{~m}$ & $1.93 \mathrm{~m}$ & $1.92 m$ & $2.04 m, 2.09 m$ \\
\hline $\begin{array}{l}7 \\
9\end{array}$ & $\begin{array}{l}1.85 m, 2.34 m \\
1.48 m\end{array}$ & $\begin{array}{l}1.83 m, 2.37 m \\
1.42 m\end{array}$ & $\begin{array}{l}1.94 m, 2.35 m \\
1.80 m\end{array}$ & $\begin{array}{l}1.92 \mathrm{~m}, 2.35 \mathrm{~m} \\
1.80 \mathrm{~m}\end{array}$ & $\begin{array}{l}1.42 m, 1.48 m \\
2.30 d(10)\end{array}$ \\
\hline 11 & $1.57 m, 1.68 m$ & $1.70 \mathrm{~m}, 1.62 \mathrm{~m}$ & $2.18 \mathrm{~m}, 2.42 \mathrm{~m}$ & $2.19 \mathrm{~m}, 2.41 \mathrm{~m}$ & $6.36 d d(16,10)$ \\
\hline 12 & $4.05 d d(10,4)$ & $4.57 d d(13,6)$ & $5.37 t(7)$ & $5.26 t(6)$ & $6.07 d(16)$ \\
\hline 14 & $5.33 t(6)$ & $5.41 t(7)$ & $6.30 d d(17,11)$ & $6.75 d d(17,11)$ & $2.19 \mathrm{~s}$ \\
\hline 15 & $4.09(2 \mathrm{H}) d(6)$ & $\begin{array}{l}3.80 d d(13,7) \\
4.05 d d(13,7)\end{array}$ & $4.87 d(11), 5.03 d(17)$ & $5.07 d(11), 5.16 d(17)$ & \\
\hline 16 & $1.59 \mathrm{~s}$ & $1.67 \mathrm{~s}$ & $1.73 \mathrm{~s}$ & $1.75 \mathrm{~s}$ & \\
\hline 17 & $4.68 s, 4.85 s$ & $4.71 s, 4.87 s$ & $4.48 s, 4.86 s$ & $4.49 s, 4.86 s$ & $2.56 d(4), 2.74 d(4)$ \\
\hline 18 & $1.17 \mathrm{~s}$ & $1.18 \mathrm{~s}$ & $1.29 s$ & $1.28 s$ & $1.26 s$ \\
\hline 20 & $0.63 s$ & $0.63 s$ & $0.64 s$ & $0.62 s$ & $0.89 s$ \\
\hline
\end{tabular}

Coupling constants $(\mathrm{J}$ in $\mathrm{Hz})$ in parentheses.

Table 2. ${ }^{13} \mathrm{C}$ NMR spectral data of compounds 12a, b, 13a, b and $16\left(\mathrm{CDCl}_{3}\right.$ solution, $\delta$ values in ppm)

\begin{tabular}{lrrrrr}
\hline $\mathrm{C}$ & $12 \mathrm{a}$ & \multicolumn{1}{c}{$12 \mathrm{~b}$} & \multicolumn{1}{c}{$13 \mathrm{a}$} & \multicolumn{1}{c}{$13 \mathrm{~b}$} & \multicolumn{1}{c}{16} \\
\hline 1 & $39.7 t$ & $40.7 t$ & $46.5 t$ & $46.4 t$ & $40.6 t$ \\
2 & $20.6 t$ & $21.6 t$ & $64.9 d$ & $64.8 d$ & $19.8 t$ \\
3 & $38.7 t$ & $39.7 t$ & $47.8 t$ & $47.7 t$ & $37.7 t$ \\
4 & $44.4 s$ & $45.5 s$ & $45.1 s$ & $45.1 s$ & $43.9 s$ \\
5 & $56.5 d$ & $57.5 d$ & $55.5 d$ & $55.4 d$ & $54.8 d$ \\
6 & $26.8 t$ & $27.8 t$ & $25.3 t$ & $25.3 t$ & $22.9 t$ \\
7 & $39.2 t$ & $40.0 t$ & $38.2 t$ & $38.2 t$ & $36.0 t$ \\
8 & $149.0 s$ & $150.8 s$ & $147.0 s$ & $146.9 s$ & $58.1 s$ \\
9 & $52.6 d$ & $53.3 d$ & $56.5 d$ & $56.4 d$ & $57.4 d$ \\
10 & $40.5 s$ & $41.5 s$ & $41.3 s$ & $41.3 s$ & $40.8 s$ \\
11 & $29.8 t$ & $30.4 t$ & $23.4 t$ & $22.3 t$ & $140.3 d$ \\
12 & $76.7 d$ & $68.2 d$ & $133.2 d$ & $130.9 d$ & $135.8 d$ \\
13 & $139.0 s$ & $139.9 s$ & $133.7 s$ & $131.9 s$ & $197.0 s$ \\
14 & $128.0 d$ & $129.0 d$ & $141.5 d$ & $133.7 d$ & $27.9 q$ \\
15 & $58.7 t$ & $59.0 t$ & $110.1 t$ & $113.5 t$ & \\
16 & $10.3 q$ & $17.0 q$ & $11.9 q$ & $19.7 q$ & \\
17 & $106.0 t$ & $106.6 t$ & $108.5 t$ & $108.7 t$ & $50.5 t$ \\
18 & $29.5 q$ & $28.4 q$ & $28.9 q$ & $28.8 q$ & $28.8 q$ \\
19 & $179.0 s$ & $179.0 s$ & $182.4 s$ & $182.5 s$ & $180.0 s$ \\
20 & $13.5 q$ & $14.4 q$ & $13.7 q$ & $13.7 q$ & $13.9 q$ \\
\hline
\end{tabular}

axial orientation indicating $S$-chirality at C-8. Related bisnorlabdanes have been found in the constituents of Juniperus thurifera [23] and Platycladus orientalis [22].

\section{EXPERIMENTAL}

Plant material. The bark of Juniperus chinensis Linn. var. kaizuka Hort. was collected from the plant grown in the surroundings of the Department of Chemistry of the
National Taiwan University. A voucher specimen is deposited in the Herbarium of our University. The airdried bark $(0.23 \mathrm{~kg})$ was soaked in $\mathrm{Me}_{2} \mathrm{CO}(51)$ for 1 week. The $\mathrm{Me}_{2} \mathrm{CO}$ extract was concd, diluted with $\mathrm{H}_{2} \mathrm{O}$ and extracted $\times 4$ with EtOAc (11 each). The combined EtOAc extracts were concd to give an oil $(20 \mathrm{~g})$, which was absorbed by $25 \mathrm{~g}$ of silica gel and then chromatographed on a column packed with $250 \mathrm{~g}$ of silica gel. Compounds 3 (69 mg), 7 (11 mg), 2 (73 mg), 6 (6 mg), 14 (4 mg), 1 (3.2 g), 4 (80 mg), 10 (2 mg), 9 (1 g), 8 (96 mg), 5 $(7 \mathrm{mg}), 15(6 \mathrm{mg}), 16(21 \mathrm{mg}), 13 \mathrm{~b}(3.5 \mathrm{mg}), 13 \mathrm{a}(10 \mathrm{mg})$ and $11(8 \mathrm{mg})$ were obtained by elution with gradients of EtOAc in hexane in ascending order of polarity $(2.5-50 \%)$, whereas $12 \mathrm{a}(7 \mathrm{mg})$ and $b(3 \mathrm{mg})$ were obtained by elution with EtOAc- $\mathrm{CHCl}_{3}$ (1:1).

Sandaracopimaric acid (1). Mp 174-175 $.[\alpha]_{\mathrm{D}}^{25}-14.4^{\circ}$ (EtOH; $c$ 1.1).

Ferruginol (2). $\mathrm{Mp} \mathrm{56-57^{ \circ }}$. $[\alpha]_{\mathrm{D}}^{25}+40.6^{\circ}\left(\mathrm{CHCl}_{3} ;\right.$ c 3.6).

7-Dehydroabietanone (3). Mp 87-90. $[\alpha]_{\mathrm{D}}^{25}+4^{\circ}$ (EtOH; c 3.4).

Sugiol (4). Mp 291-293 ${ }^{\circ}[\alpha]_{\mathrm{D}}^{25}+25^{\circ}$ (EtOH; $c$ 8).

$6 \alpha-H y d r o x y-7$-oxoferruginol (5). Mp 207-208 ${ }^{\circ}[\alpha]_{\mathrm{D}}^{25}$ $+35.3^{\circ}$ (MeOH; $c 0.33$ ).

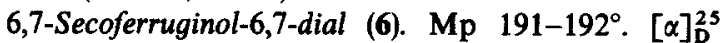
$+20^{\circ}$ (MeOH; $\left.c 0.2\right)$.

Totarol (7). Mp 131-132 ${ }^{\circ}[\alpha]_{\mathrm{D}}^{25}+40.2^{\circ}$ (EtOH; c 2.2). Totarolone (8). Mp $188-189^{\circ} .[\alpha]_{\mathrm{D}}^{25}+101.5^{\circ}$ (MeOH; c 3.2).

Communic acid (9, a mixture of $12 E$ - and $12 Z$-isomers, 3:1). Mp 130-132 $.[\alpha]_{\mathrm{D}}^{25}+38^{\circ}$ (EtOH; $c$ 1.0).

Isocupressic acid (10). Mp $117-120^{\circ} .[\alpha]_{\mathrm{D}}^{25}+14^{\circ}$ (EtOH; $c$ 1.5).

Agathic acid (11). Mp 203-204. $[\alpha]_{\mathrm{D}}^{25}+58^{\circ}\left(\mathrm{CHCl}_{3}\right.$; $c 0.8$ ). 
12,15-Dihydroxylabda-8(17),13E-dien-19-oic acid (12a). Solid, mp $178 \cdots 180^{\circ} .[\alpha]_{\mathrm{D}}^{25}+33.3^{\circ}(\mathrm{EtOH} ; c$ 0.4). IR $v_{\max }^{\mathrm{KBr}} \mathrm{cm}^{-1}: 3500-2500,1690\left(\mathrm{CO}_{2} \mathrm{H}\right)$. EIMS $(70 \mathrm{eV}) \mathrm{m} / \mathrm{z}$ (rel. int.) $318\left[\mathrm{M}-\mathrm{H}_{2} \mathrm{O}^{+}\right.$(4), $300(18), 285(21), 235$ (17), $221(19), 207(11), 189(50), 121(80), 81(80), 55(100)$. Exact mass for $\mathrm{C}_{20} \mathrm{H}_{30} \mathrm{O}_{3},\left[\mathrm{M}-\mathrm{H}_{2} \mathrm{O}\right]^{+}$, requires 318.2196 . Found 318.2206.

12,15-Dihydroxylabda-8(17),13Z-dien-19-oic acid (12b). Solid, mp 186-188. $[\alpha]_{\mathrm{D}}^{25}+9^{\circ}(\mathrm{MeOH} ; c 0.45)$. IR $v_{\max }^{\mathrm{KBr}} \mathrm{cm}^{-1}: 3500-2500,1690\left(\mathrm{CO}_{2} \mathrm{H}\right), 1630,1600,882$. EIMS $(70 \mathrm{eV}) \mathrm{m} / \mathrm{z}$ (rel. int.) $336\left[^{[\mathrm{M}]^{+}}\right.$(2), $318[\mathrm{M}$ $\left.-\mathrm{H}_{2} \mathrm{O}\right]^{+}$(16), 300 (28), 285 (33), 235 (38), 189 (90), 121 (87), 83 (100).

2 $\alpha$-Hydroxy-trans-communic acid (13a). Solid, mp 121-122. $[\alpha]_{\mathrm{D}}^{25}+16.6^{\circ}(\mathrm{MeOH} ; c 0.2)$. IR $v_{\max }^{\text {neal }} \mathrm{cm}^{-1}$. $3600-3200,1688\left(\mathrm{CO}_{2} \mathrm{H}\right), 1640,1026,910$. UV $\lambda_{\max }^{\mathrm{MeOH}} \mathrm{nm}$ (e): $227(3600)$. EIMS $(70 \mathrm{eV}) \mathrm{m} / z$ (rel. int.) $318[\mathrm{M}]^{+}(18)$, $300\left[\mathrm{M}-\mathrm{H}_{2} \mathrm{O}\right]^{+}(20), 285(12), 255(19), 199(20), 187$ (26), $173(67), 79(100), 55$ (64). Exact mass for $\mathrm{C}_{20} \mathrm{H}_{30} \mathrm{O}_{3}$, $\left[\mathrm{M}-\mathrm{H}_{2} \mathrm{O}\right]^{+}$, requires 318.2196 . Found 318.2184.

$2 \alpha$-Hydroxy-cis-communic acid (13b). Solid, $\mathrm{mp}$ $132133^{\circ} \cdot[x]_{\mathrm{D}}^{25}+18.7^{\circ}\left(\mathrm{CHCl}_{3}, c 0.35\right) . I R v_{\max }^{\text {neat }} \mathrm{cm}^{-1}$ : $3600-3200,1682\left(\mathrm{CO}_{2} \mathrm{H}\right), 1640,988,890 . \mathrm{UV} \lambda_{\max }^{\mathrm{CHCl}_{3}} \mathrm{~nm}$ (ع): 245 (1600). EIMS (70 eV) $m / z$ (rel. int.) $318\left[\mathrm{M}^{+}{ }^{+}(29)\right.$, 300 (33), 285 (24), 255 (37), 199 (26), 187 (28), 173 (80), 79 (100), $55(62)$. Exact mass $[\mathrm{M}]^{+}$for $\mathrm{C}_{20} \mathrm{H}_{30} \mathrm{O}_{3}$ requires 318.2196. Found 318.2184.

12,13-Epoxylabda-8(17),14-dien-19-oic acid (14). Oil. $[\alpha]_{\mathrm{D}}^{25}+24^{\circ}\left(\mathrm{CHCl}_{3} ; c 0.2\right) .{ }^{1} \mathrm{H} \mathrm{NMR}\left(\mathrm{CDCl}_{3}\right): \delta 0.56(3 \mathrm{H}$, $s, \mathrm{H}-20), 1.22$ (3H, $s, \mathrm{H}-18), 1.36(3 \mathrm{H}, s, \mathrm{H}-16), 2.87$ (dd, $J$ $=6,4 \mathrm{~Hz}, \mathrm{H}-12), 4.45(s, \mathrm{H}-17), 4.84(s, \mathrm{H}-17), 5.27(d d, J$ $=10,1.4 \mathrm{~Hz}, \mathrm{H}-15), 5.29(d d, J=17,1.4 \mathrm{~Hz}, \mathrm{H}-15), 5.78$ $(d d, J=17,10 \mathrm{~Hz}, \mathrm{H}-14)$. Exact mass $[\mathrm{M}]^{+}$for $\mathrm{C}_{20} \mathrm{H}_{30} \mathrm{O}_{3}$ requires 318.2196 . Found 318.2182 .

12,13-Dihydroxylabda-8(17),14-dien-19-oic acid. (12R, $13 S)$-Isomer $15 \mathbf{a}$, oil, $[\alpha]_{\mathrm{D}}^{25}+18^{\circ}\left(\mathrm{CHCl}_{3} ;\right.$ c 0.7$)$. ${ }^{1} \mathrm{H} \mathrm{NMR}\left(\mathrm{CDCl}_{3}\right): \delta 0.58(3 \mathrm{H}, s), 1.22(3 \mathrm{H}, s, \mathrm{H}-18), 1.25$ $(3 \mathrm{H}, s, \mathrm{H}-16), 3.48(1 \mathrm{H}, d, J=10 \mathrm{~Hz}, \mathrm{H}-12), 4.42(1 \mathrm{H}, s, \mathrm{H}-$ 17), $4.82(1 \mathrm{H}, \mathrm{s}, \mathrm{H}-17), 5.19(1 \mathrm{H}, d d J=10,1 \mathrm{~Hz}, \mathrm{H}-15)$, $5.33(1 \mathrm{H}, d d, J=17,1 \mathrm{~Hz}, \mathrm{H}-15), 5.91(1 \mathrm{H}, d d, J=17$, $10 \mathrm{~Hz}, \mathrm{H}-14) .{ }^{13} \mathrm{C} \mathrm{NMR}\left(\mathrm{CDCl}_{3}\right): \delta 12.5(\mathrm{C}-20), 19.8(\mathrm{C}-2)$, 23.1 (C-11), 25.0 (C-18), 26.1 (C-6), 28.7 (C-16), 38.3 (C-3), 38.5 (C-7), 39.3 (C-1), 39.9 (C-10), 44.3 (C-4), 51.9 (C-9), 56.3 (C-5), 75.3 (C-13), 77.5 (C-12), 106.8 (C-17), 114.2 (C-15), 141.1 (C-14), 147.8 (C-8), 177.6 (C-19). Exact mass for $\mathrm{C}_{20} \mathrm{H}_{30} \mathrm{O}_{3}\left[\mathrm{M}-\mathrm{H}_{2} \mathrm{O}\right]^{+}$requires 318.2196. Found 318.2202. (12R,13R)-Isomer 15b, ${ }^{1} \mathrm{H} \mathrm{NMR}\left(\mathrm{CDCl}_{3}\right.$ inter alia): $\delta 1.30(3 \mathrm{H}, s, \mathrm{H}-16), 3.45(1 \mathrm{H}, d, J=10 \mathrm{~Hz}, \mathrm{H}-12)$, $4.43(1 \mathrm{H}, s, \mathrm{H}-17), 4.84(1 \mathrm{H}, s, \mathrm{H}-17), 5.20(1 \mathrm{H}, d, J$ $=10 \mathrm{~Hz}, \mathrm{H}-15), 5.33(1 \mathrm{H}, d, J=17 \mathrm{~Hz}, \mathrm{H}-15), 5.91(1 \mathrm{H}, d d$, $J=17, \quad 10 \mathrm{~Hz}, \quad$ II-14). (12S,13RS)-Isomers, ${ }^{1} \mathrm{H}$ NMR $\left(\mathrm{CDCl}_{3}\right.$, inter alia): $\delta 3.38 / 3.39(d, J=10 \mathrm{~Hz}, \mathrm{H}-12), 4.75(s$, $\mathrm{H}-17), 4.89$ ( $s, \mathrm{H}-17), 5.17$ ( $d, J=10 \mathrm{~Hz}, \mathrm{H}-15), 5.33$ ( $d, J$ $=17 \mathrm{~Hz}, \mathrm{H}-15), 5.90$ (dd, $J=17,10 \mathrm{~Hz}, \mathrm{H}-14$ ).

15,16-Bisnor-8,17-epoxy-13-oxolabd-11 E-en-19-oic acid (16). Oil, $[\alpha]_{\mathrm{D}}^{25}+7^{2}\left(\mathrm{CHCl}_{3} ; c 1.0\right)$. IR $\nu_{\max }^{\text {ncat }} \mathrm{cm}^{-1}$ : $3600-3200,1700(\mathrm{C}=\mathrm{O}), 1688,1640,1252,1000,890$. UV $\lambda_{\max }^{\mathrm{MeOH}} \mathrm{nm}(\varepsilon): 227(1833)$. EIMS (70 eV) $\mathrm{m} / z$ (rel. int.): 306
$[\mathrm{M}]^{+}(3), 275(50), 263$ (10), 167 (9), 121 (41), 55 (36), 43 (100). Exact mass $[\mathrm{M}]^{+}$for $\mathrm{C}_{18} \mathrm{H}_{26} \mathrm{O}_{4}$ requires 306.1831 . Found 306.1823.

Acknowledgement-We are grateful to the National Science Council of the Republic of China for financial support.

\section{REFERENCES}

1. Ying, S. S. (1985) Coloured Illustrated Flora of Taiwan Vol. 1, p. 168. National Taiwan University, Taipei.

2. Hsu, C. M. (1960) Chemical Constituents of the Medicinal Plants in Taiwan Vol. 1, 174. National Research Institute of Chinese Medicine, Taipei.

3. Sawada, T. (1958) Yakugaku Zasshi 78, 1020

4. Fang, J. M., Lee, C. K. and Cheng, Y. S. (1992) Phytochemistry 31, 3659.

5. Fang, J. M., Lee, C. K. and Cheng, Y. S. (1993) Phytochemistry (in press).

6. Edwards, O. E., Nicolson, A. and Rodger, M. N. (1960) Can. J. Chem. 38, 663.

7. Fang, J. M., Sheu, C. M. and Cheng, Y. S. (1986) J. Chin. Chem. Soc. (Taipei) 33, 245.

8. Nishida, T., Wahlberg, I. and Enzell, C. R. (1977) Org. Magn. Res. 9, 203.

9. Defaye-Duchateau, G. (1964) Bull. Soc. Chim. Fr. 1496.

10. Kuo, Y. H., Chang, B. H. and Lin, Y. T. (1975) J. Chin. Chem. Soc. (Taipei) 21, 19.

11. Fang, J. M., Jan, S. T. and Cheng, Y. S. (1986) J. Chem. Res. (S) 350 .

12. Cambie, R. C. and Mander, L. N. (1962) Tetrahedron 18, 465.

13. Chow, Y.-L. and Erdtman, H. (1960) Acta Chem. Scand. 14, 1852.

14. Bohlmann, F. and Zdero, C. (1974) Chem. Ber. 107, 1416.

15. Fang, J. M., Hsu, K. C. and Cheng, Y. S. (1989) Phytochemistry 28, 1173.

16. Fang, J. M., Lang, C. I., Chen, W. L. and Cheng, Y. S. (1991) Phytochemistry 30, 2793.

17. Carman, R. M. (1964) Aust. J. Chem. 17, 393.

18. Caputo, R. and Mangoni, L. (1974) Phytochemistry 13, 467.

19. Fujimoto, Y., Kakinuma, K., Eguchi, T., Ikekawa, N., Hirayama, N., Mbarushimana, A. and Ntahomvukiye, D. (1990) Phytochemistry 29, 319.

20. Cambie, R. C., Grigor, B. A. and Tiong, M. I. (1981) Aust. J. Chem. 34, 1073.

21. De Pascual Teresa, J., San Feliciano, A., Tabernero, M. L., Del Corral, J. M. M., Barrero, A. F. and Grande, M. (1978) An. Quim. 74, 459.

22. Inoue, M., Hasegawa, S. and Hirose, Y. (1985) Phytochemistry 24, 1602.

23. De Pascual Teresa, J., San Feliciano, A. and Egido, T. (1976) An. Quim. 72, 865 